\title{
Non Glycaemic Effects of Sitagliptin - Systematic Review of Six Randomised Controlled Clinical Trials
}

Authors: Vipul Gupta1, Girish Khurana², Anuradha Gupta ${ }^{1}$, Archana Khurana², Hardik Khurana ${ }^{2}$, Navneet Wadhwa ${ }^{3}$

\section{Institution}

1. Gupta Ultrasound and Heart Care Centre, Delhi

2. Vidya Medicare Centre, Haryana

3. AUW Global, Delhi

\section{Background}

- DPP-4 inhibition using sitagliptin has been a novel concept for the management of T2D

- It has been well established that Sitagliptin is efficacious and plays an important role in the management of T2DM, both in combination and monotherapy

- In the current therapeutic evolution of gliptins it is important to understand the systemic effects of DPP IV inhibition by sitagliptin

\section{Aim}

- To explore the evidence for the contemporary role of sitagliptin in the current evolution of evidence base for the gliptins and understand and evaluate for the non glycemic effects of sitagliptin, through analysing the quantity and quality of the published evidences through the highest level of evidences ie: Randomised Controlled Clinical Trials (RCCTs)

\section{Method}

- We searched Cochrane Library, pubmed- MEDLINE, IndMED online databases to conduct a systematic review of the published Randomised Controlled Clinical Trials (RCCTs) evaluating the contemporary role of sitaglitin for the non-glycemic effects

- Only the trials with the comparator arms were included for our analysis and the studies evaluating the glycemic effects of sitaglitin were excluded by using the appropriate Boolean operators.

- Only the RCCTs which included the health subjects and non-diabetics were include

- Graph pad prism 7.0 version software and t-test was utilised for statistical analysis.

\section{Results}

- 295 clinical trials have been published which have explored the benefits of Sitagliptin

- The results yielded 8 RCCTs of which six met the inclusion criteria, which are published in last 9 years (2008 to 2016)

- Only the RCCTs with comparator arms were included for the analysis

- The parameters were analysed for the study design, patient characteristics, geography of the study, impact factor of the journals, intervention, duration and outcomes by using the appropriate statistical methods

- Cumulatively, 269 patients (mean 45 patients, $S D \pm 64$, SEM \pm 26 , minimum 6 patients, maximum 174 patients, $95 \% \mathrm{CI}-23$ to $112, \mathrm{p}=0.147$ ) have been evaluated across 6 RCCTs. (Fig 1 \& 2)

- The duration of the RCCTs varies from 1 day to 1 year $(\mathrm{n}=2)$

- USA is the predominant country which contributes to the evidence $(n=4)$ followed by one each from Germany, Switzerland and Taiwan

\author{
NOVEL
}

NOVEL
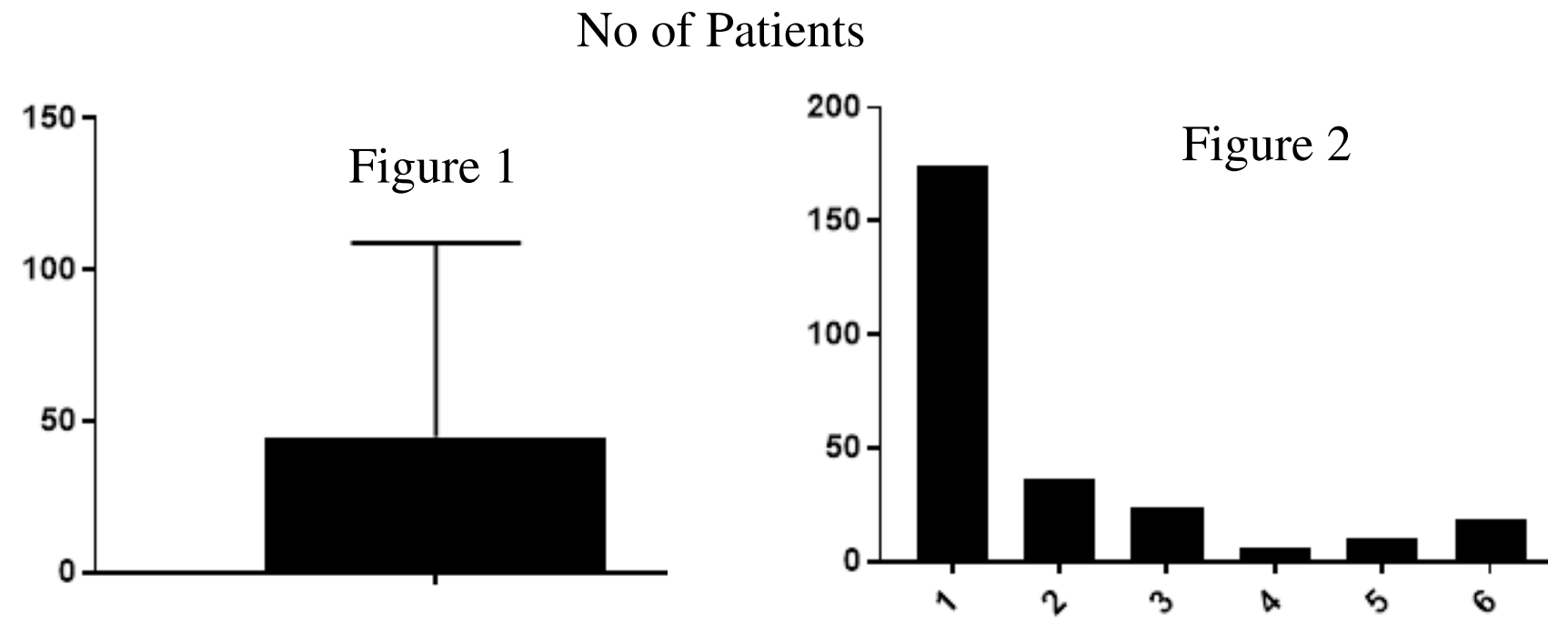

- Based on the impact factor of the journals (mean 3.5, minimum 1.5, maximum 5.5, $\mathrm{SD} \pm 1.4, \mathrm{SEM} \pm 0.59,95 \% \mathrm{CI} 2$ to 5.1 ; $\mathrm{p}=0.0019$ ), (Fig $3 \& 4$ ) we formulated an indexed weightage score (mean 100, minimum 43.34, maximum 156.4, SD $\pm 40.9, \mathrm{SEM} \pm$ $16.7,95 \%$ CI 57.08 to 142.9 ) (Fig $5 \& 6$ )
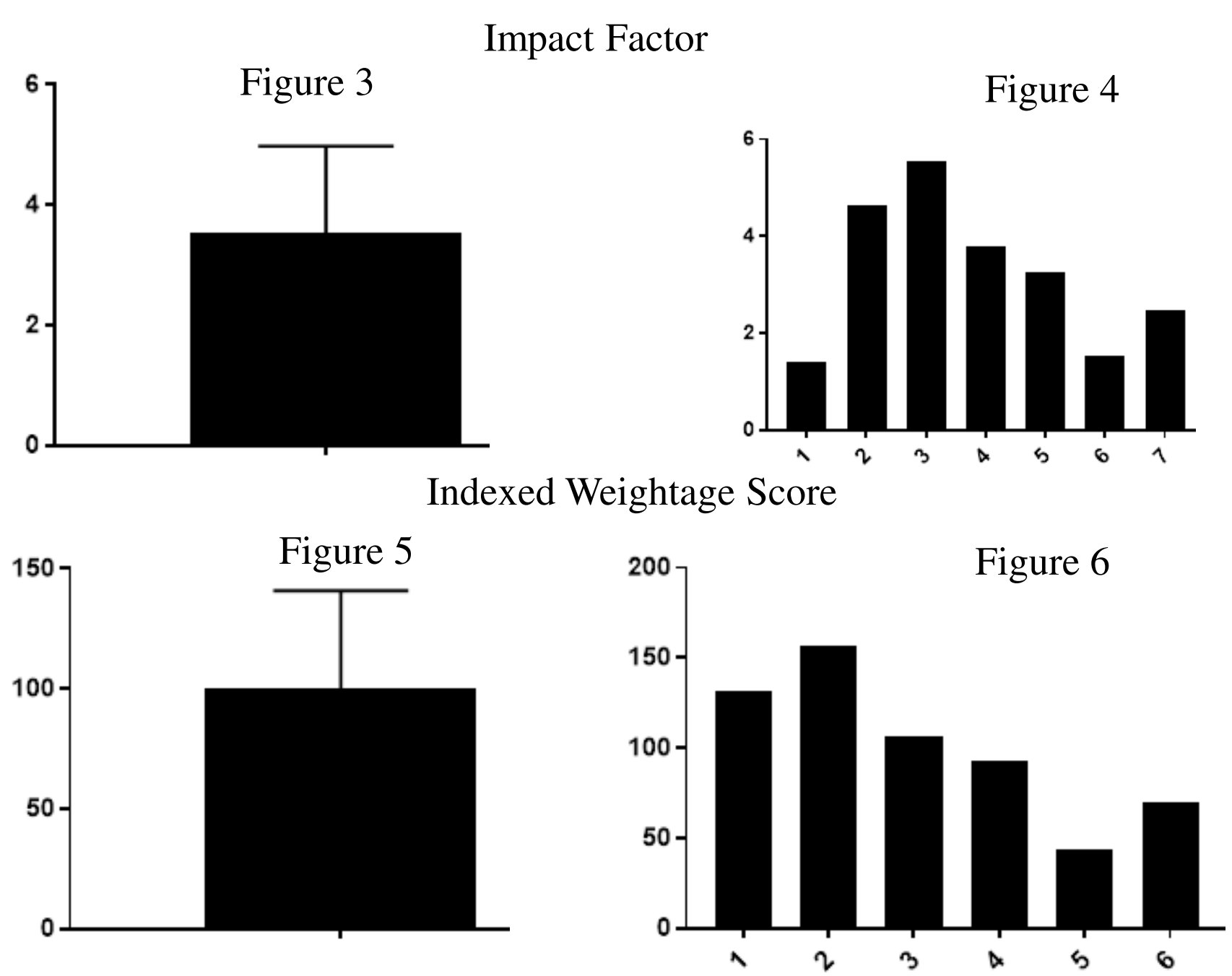

- The Journal of Clinical Endocrinology and Metabolism (Impact Factor of 5.531) is the highest impact factor journal which revealed the benefits of sitagliptin in systemic and adipose antiinflammatory effects in cART-treated HIV+ adults with impaired glucose tolerance

- The RCCTs demonstrate the benefits for the improvement in the left and right ventricular ejection fraction changes from baseline to 6 months of follow-up in combination with Sitagliptin plus granulocyte colony-stimulating factor in patients suffering from acute myocardial infarction (SITAGRAMI trial)

- Results favour for the role in control of hypertension, single-unit cord blood transplants in adults with hematological malignancies, neutral effect on the circulating ghrelin and gastrin levels

\section{Discussion}

- The evaluation of the contemporary published evidences reveal that sitagliptin is useful in varied clinical conditions with nonglycemic effects.

- There is a scope to expand the utility of this novel gliptin by validating the results across global multicentric trials which would yield large scale mechanistic insights across varied non diabetic population 